\title{
Analysis of cotton height spatial variability based on UAV-LiDAR
}

\author{
Kuan Liu, Xiaoya Dong, Baijing Qiu* \\ (Key Laboratory of Crop Protection Engineering, Ministry of Agriculture and Rural Affairs, Jiangsu University Zhenjiang, 212013, China)
}

\begin{abstract}
The spatial variance of geometric information of farmland crops is the basis of field management. Therefore, it has significance for variable mechanical operations to accurately obtain the spatial difference of crop height information. In the present study, UAV-LiDAR was used to collect data at the cotton planting base in Korla to estimate the spatial differences in cotton plant height. The crop height was estimated using the average height of a certain number of highest points per $\mathrm{m}^{2}$ point cloud. First, the plant heights of different spatial locations in the field were collected manually and compared with the system measurement. The results showed that the maximum relative error of sampling was $12.73 \%$, the error value was $3.48 \mathrm{~cm}$, and the height map was visualized. In order to explain the height change of plant height in the direction of crop rows and vertical crop rows, this paper used the coefficient of variation as a measure. The results showed that the plant height variation coefficient in the crop row direction ranged from 0.54-1.04 and the average variation coefficient was 0.73; perpendicular to the crop row direction, the crop height variation coefficient range was 0.06-1.27 and the average variation coefficient was 0.58 . The spatial difference information was characterized by the coefficient of variation of the geometrical features of the crop height. This work can provide information for cotton field variable operation machinery and provide reference for the extraction of field crop geometric information.
\end{abstract}

Keywords: LiDAR, plant parameter, spatial variability, plant height

DOI: $10.33440 /$ j.ijpaa.20200303.79

Citation: Liu K, Dong X Y, Qiu B J. Analysis of cotton height spatial variability based on UAV-LiDAR. Int J Precis Agric Aviat, 2020; 3(3): 72-76.

\section{Introduction}

Crop height information is an important basis for adjusting farmland management measures. Farmland management includes the regulation of soil moisture, nutrition, pests, etc. These conditions will affect the growth of crops, mainly reflected by changes in crop morphological characteristics ${ }^{[1]}$, physical characteristics $^{[2]}$ and physiological characteristics ${ }^{[3,4]}$. The crop heights are different in different areas of the cotton field, so the acquisition of crop characteristics information has become the information base for adjusting farmland management strategies.

Remote sensing is a non-intrusive detection technology, and a large number of scholars have conducted researches on different traits of different crops ${ }^{[5-7]}$. Spectral technology can be used to reflect spectrum data to estimate crop growth, especially to obtain physiological and physical characteristics such as crop leaf moisture content ${ }^{[8]}$, chlorophyll content ${ }^{[9]}$, nitrogen concentration $^{[10]}$, and height. Similarly, thermal imaging technology can detect the heat emitted by objects. The temperature of the crop canopy can be detected by thermal imaging, and it has developed into an important indicator to reflect the moisture content of crops $^{[11]}$. The LiDAR (Light Detect and ranging) sensor estimates the distance between the sensor and the target crop by recording the laser time of flight (TOF) ${ }^{[12]}$. The data output of LiDAR sensor can be used to reconstruct 3D point

\section{Received date: 2020-06-03 Accepted date: 2020-09-17}

Biographies: Kuan Liu, Graduate student, research interests: precision application, variable spraying, Email: 22111616020@ujs.edu.cn; Xiaoya Dong, $\mathrm{PhD}$, Associate Professor, research interests: agricultural aerial application technology, Email: dongxiaoya@ujs.edu.cn;

*Corresponding author: Baijing Qiu, PhD, Professor, research interests: precision agricultural aviation application, Jiangsu University, Xuefu Road Jingkou District, Zhenjiang City, Zhenjiang, 212013, China. Email: qbj@ujs.edu.cn. clouds to obtain the morphological characteristics of crops. The 3D point cloud reconstructed by LiDAR sensor provides high-precision characterization of canopy volume and crop density ${ }^{[13]}$.

LiDAR, as a new technology developed in the past ten years, has been proved to apply for the geometric information detection of crops. It has the characteristics of high accuracy and little influence by light ${ }^{[14-16]}$. When LiDAR was early used in crop detection, LiDAR was carried on a ground-based mobile device, and the scanning path had to maintain a fixed distance from the tested crop. Polo et al. ${ }^{[17]}$ developed a LIDAR system mounted on a tractor platform to quickly and non-destructively estimate plant volume and leaf area characteristics. In the same year, Rosell et al. ${ }^{[18]}$ optimized the registration of crop data with the help of computer-aided design (CAD) software and demonstrated that the 3D point cloud plant structure was consistent with the real structure, and the highest volume correlation coefficient was 0.976. With the help of GNSS information 3D point cloud data, the local coordinate system is synchronized to the geographic coordinate system, so that the data collection does not need to maintain a fixed distance from the tree row, the point cloud data on both sides of the tree row can be accurately matched, and the processing has been greatly improved $^{[19]}$. Colaço et al. ${ }^{[20]}$ developed a method of collecting and analyzing point cloud data containing geographic information, which can realize the morphological characteristics of crops in large-scale orange orchard environments and realize the visualization of the spatial variability of features. With the development of unmanned aerial vehicle technology, the LiDAR system using drones as the platform has been rapidly applied due to the characteristics of detection without the limitation of line distance, soil moisture and easy control. It is worth noting that UAV measurement methods also have limitations. For example, Lei et al. ${ }^{[21]}$ studied the relationship between the position of the drone relative to the measured crop and the density of the crop. 
The LiDAR measurement system mounted on the UAV improves the efficiency of information acquisition and the scale. The amount of data also increased rapidly, including a large amount of point cloud data of farmland and the surrounding environment. Therefore, the extraction of crop characteristics in point cloud data has become the focus of research.

Researches on plant morphological estimation can be classified according to the number of plants. The phenotype of a single plant, the crops are mostly in the seedling stage with small planting density, and the point cloud data of a single plant can be extracted to estimate the plant morphological parameters. Deep learning can be used to identify individual crops. Jin et al. ${ }^{[22]}$ compressed the corn plant point cloud into a depth map as a training set, used the Faster R-CNN (region-based convolutional neural network) model to learn the ability to detect corn stalks, and then mapped the $3 \mathrm{D}$ points of the test set to be used as the seed point of the regional growth algorithm to detect the individual plant corn. The results obtained by the measuring system and manual method to measure the height of corn has a good correlation $\left(R^{2}>0.9\right)$. More scholars focused on extracting geometric feature parameters from multiple point clouds ${ }^{[23-26]}$. Yuan et al. developed a phenotype measurement system for peanut canopy based on a bicycle platform and proposed a method for dividing a single row of peanut canopy according to extreme points and calculating the canopy height from the highest point of the canopy. There is a strong correlation $\left(R^{2}=0.915\right)$ between the canopy height obtained by the system and the manually measured value. The root mean square error is $22.78 \mathrm{~mm}^{[27]}$. Ashapure et al. ${ }^{[28]}$ extracted the geometric characteristics of crop growth by dividing the point cloud data of cotton fields into 1 square meter grids. By comparing the differences in canopy height, coverage and canopy volume, it was proved that reducing farming could improve crop geometric parameters. Colaço et al. ${ }^{[29]}$ obtained the canopy height and volume of the orange garden through point cloud data, proving the spatial variability of the canopy geometry. Among them, the coefficient of variation of the canopy volume was $30 \%$ to $40 \%$, and the information can be used in agricultural machinery variable assignments. In this paper, the method of obtaining geometric parameters of cotton plants from point cloud data in high-density cotton fields was studied, and the spatial differences in cotton plant heights were calculated. Firstly, the location of the experiment and the growth of cotton plants were introduced. Secondly, the point cloud data preprocessing method and the calculation method of cotton plant height were introduced. Finally, the spatial difference of cotton plant height was counted and the difference map was drawn.

\section{Materials and methods}

\subsection{Experimental site}

The climate of Korla, Xinjiang, China is a typical temperate continental arid climate. The annual average temperature is around $11^{\circ} \mathrm{C}$, and the average annual precipitation is less than $60 \mathrm{~mm}$. It is suitable for cotton planting, with a large planting scale and a high degree of mechanization. It is an important part of China's largest cotton producing area. In this study, the scientific research base of the Plant Protection Institute of Xinjiang Academy of Agricultural Sciences $\left(41^{\circ} 1^{\prime} 55^{\prime \prime N}, 85^{\circ} 48^{\prime} 18^{\prime \prime E}\right)$ in Korla was selected as our research area. The measurement object is cotton (Xinluzhong66) in the peach-making period, with planting density of $15-18$ plants $/ \mathrm{m}^{2}, 17-19$ leaves, average plant height of cotton $50-55 \mathrm{~cm}$, and leaf area index of 1.556. The growth of cotton plants is shown in Figure 1.

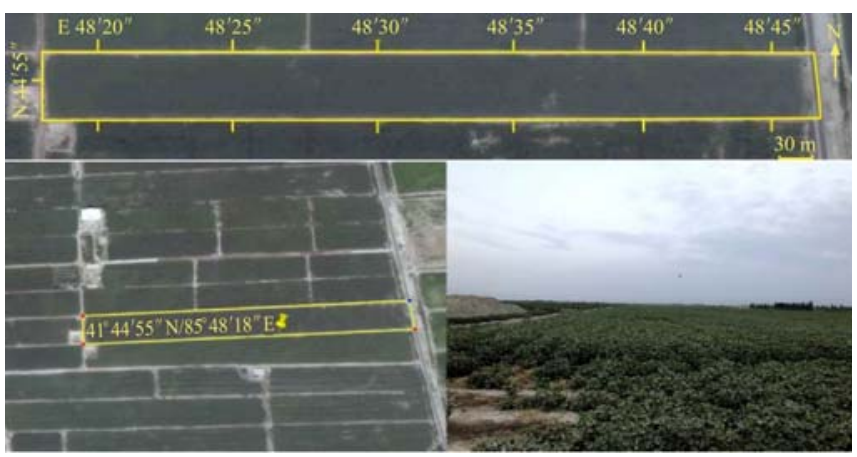

Figure 1 Cotton plants

\subsection{Data acquisition}

\subsubsection{Hardware equipment}

A three-dimensional lidar crop phenotype detection system (abbreviated as a detection system) was used to collect geometric information of cotton plants. The detection system is a portable lidar measurement system, which mainly integrates a 16-line laser scanner, GNSS using post-processing technology, inertial navigation system INS and control memory. The main parameters of the system are shown in Table 1. The system is mounted on the plant protection UAV platform (DJI MG-1P). To ensure the measurement accuracy, the UAV platform needs to fly around to make the GNSS accuracy converge. The stationary state of the crop has changed by the wind field of UAV, causing measurement errors. In order to eliminate the influence of the wind field, the flying height of the UAV platform in this paper is not less than $9 \mathrm{~m}$.

Table 1 VLP-16 and SPAN-IGM-A1 parameters

\begin{tabular}{clc}
\hline \multicolumn{1}{c}{ Sensor } & \multicolumn{1}{c}{ Specification } & Value \\
\hline & Channels & 16 \\
& Measurement Range/m & $<100$ \\
& Range Accuracy/cm & \pm 3 \\
Velodyne & Angular Resolution (Vertical) $/\left(^{\circ}\right)$ & 2 \\
VLP-16 & Angular Resolution (Horizontal) $/\left(^{\circ}\right)$ & $0.1-0.4$ \\
& Rotation Rate/Hz & $5-20$ \\
& Weight/g & 830 \\
\hline & Single point L1/L2(RMS)/m & 1.2 \\
& RTK (RMS) & $1 \mathrm{~cm}+1 \mathrm{ppm}$ \\
& GNSS Data Rates/Hz & 20 \\
& IMU Data Rates/Hz & 200 \\
& Weight/g & 515 \\
\hline
\end{tabular}

The detection system is designed to efficiently obtain field phenotype data. The 3D laser scanner can have a vertical angle resolution of $2^{\circ}$, a horizontal angle resolution of $0.1-0.4^{\circ}$, a vertical field of view of $30^{\circ}$, a horizontal field of view of $360^{\circ}$, a measurement frequency of $5-20 \mathrm{~Hz}$, and a maximum measurement distance of $100 \mathrm{~m}$. RTK-GNSS can provide positioning data with an accuracy of $10 \mathrm{~cm}$ and a measurement frequency of $20 \mathrm{~Hz}$.

\subsubsection{Field experiments}

The field data collection test time was August 15, 2019. During the data acquisition period, to avoid the interference of strong sunlight on LiDAR working temperature and signals in high latitudes, the test was selected in the evening, and the environmental wind degree was less than $0.87 \mathrm{~m} / \mathrm{s}$. The detection system was mounted on the UAV platform and travels at a uniform speed of $2 \mathrm{~m} / \mathrm{s}$ in the direction of the cotton ridge, and the vertical height from the ground was $9 \mathrm{~m}$. The maximum value of the obtained point cloud data density was 13000 points $/ \mathrm{m}^{2}$ and the 
average value was 1681.94 points $/ \mathrm{m}^{2}$. The cotton plant height was divided into three gradients: high, medium, and low. Three crops were randomly selected for each gradient, and the average value was obtained by manual measurement and comparison with the measurement data of the LiDAR system.

\subsection{Data Processing}

After acquiring the raw data of cotton fields in the field, the data processing and analysis were performed in matlab2019b (the math works Inc., Natic, MA, USA) software, the operating system platform is Ubuntu16.04, and the computer configuration is I9-9900k CPU $3.60 \mathrm{GHz}$ 16GB RAM. To extract the target field and the geometric parameters of the cotton plant from the original high-density point cloud data, the data analysis and processing program was mainly divided into three steps: original data preprocessing, target field block extraction and crop geometric parameter extraction.

\subsubsection{Data Pre-Processing}

Due to the raw point cloud data which containing a large number of three-dimensional points and existing of data redundancy, the detection accuracy of crops was reduced and the data processing time was increased. Outliers will cause a decrease in data processing accuracy. This article preprocesses the original data by down-sampling and KD-Tree denoising.

1) Point cloud thinning. In order to reduce redundant data and keep the field sample data independent and identically distributed with the original data, this article uses random sampling method $^{[30]}$. The point clouds data were divided into multiple small cube grids, and a point is randomly selected in each grid as the down-sampling value. The number of points was reduced from 12.67 million points to 9.32 million points, and the data compression ratio was $26.44 \%$.

2) Denoising the point cloud. In view of the random distribution of cotton field point cloud data, dense data, and large altitude changes, a method based on average neighborhood distance was selected. Constructed a k-d tree, established a point cloud topological relationship in the target area, calculate the average distance $d$ and standard deviation $\sigma$ of each point from its nearest neighbor point, and the neighbor parameter $k_{d}$. If $\mathrm{d}$ exceeds the set threshold $d_{\text {max }}$, the point is determined as a noise point and deleted. In this paper, the $\mathrm{k}$ - $\mathrm{d}$ tree algorithm parameter value is $k_{d}=10$, and the threshold value $d_{\max }=12$.

\subsubsection{Target field extraction}

The original file of point cloud data is in LAS format, which contains objects such as crops, buildings and trees. In order to extract crop point clouds, the original point clouds are cropped.

1) Reading the LAS file.

The point cloud coordinate values were the representation in the Gaussian plane coordinate system. The location of the test field has a large coordinate value in Gaussian coordinate system. In order to improve the calculation speed and accuracy, the point cloud coordinates were subtracted from the point cloud center of gravity coordinates. The center of gravity of a point cloud was a $3 \mathrm{D}$ point coordinate, and the coordinate value is the average value of all points in the point cloud. In this paper, the barycenter coordinates of point cloud was $(400640.77 \mathrm{~m}, 4622524.04 \mathrm{~m}$, $840.11 \mathrm{~m})$.

2) Extracting target point cloud.

Usually the direction of the crop row in the point cloud was different from the direction of the graphic coordinate axis. Therefore, it is necessary to eliminate the angle by rotating the point cloud. Then, Setting the value range (x_min $\left.x \_m a x\right)$, (y_min $\left.y \_m a x\right),\left(z \_m i n ~ z \_m a x\right)$ on the coordinate axis to complete the cutting. The field coordinate system is established using the principal component analysis method (PCA) ${ }^{[31]}$.

\subsubsection{Cotton plant height map}

Plant height can be defined as the distance from the ground to the top of the canopy. In the measurement process using laser sensors, due to various factors such as different environments, ground undulations and crop growth stages, the method of extracting crops from point cloud data is very different. The data used in this paper has small local fluctuations in the ground and dense crop point clouds. At the same time, because the probability of the laser beam of the sensor passing through the crop canopy was small, the number of ground point clouds acquired was small. Under the condition of high planting density, it is difficult to extract the point cloud of single crop. In this paper, the point cloud was divided into regions, and the regional height was used to represent the height of crop groups. This is more conducive to the application in variable machines. Due to differences in crop canopy structure, the highest point in the point cloud is not always the best representative of the crop height measured in the field. Therefore, for each point cloud grid area, the highest 5 points were selected to average to represented the height of crop groups in this region.

\subsubsection{Coefficient of variation}

The coefficient of variation of the plant height in cotton fields can be analyzed from the crop row and its vertical direction from two dimensions. In this paper, the point cloud data is projected along the crop row at $1 \mathrm{~m}$ intervals, and the data is projected along the crop row, and the plant height is calculated the coefficient of variation in this direction, the sample coefficient of variation (CV) is defined as the ratio of the standard deviation to the mean:

$$
c v=\frac{s}{\bar{x}}
$$

where, $s$ is the sample standard deviation and $\bar{x}$ is the sample mean. It shows the variability relative to the mean defined by the standard deviation.

\section{Results and discussion}

\subsection{Cotton plant height measurement results and analysis}

To determine the accuracy of the measured data in the experiment, A comparison between the LiDAR analysis results of the cotton canopy average height and the manual measurement values are shown in Table 2 . The results clearly show that the system measurement values were lower than the manual measurement values, the maximum relative error of sampling is $12.82 \%$, and the corresponding maximum sampling error is $3.48 \mathrm{~cm}$. The main reason for this result was that the sampling resolution of the laser sensor was $3 \mathrm{~cm}$. When the laser sensor measured the height of the crop, the size of the branches and leaves at the top of the crop were small, which caused the measurement error of the sensor to increasing, and even the top of the crop cannot be measured. In manual measurement, the position of the top of the crop can been easily judged, so the manual measurement of cotton plant height is greater than the system measurement value. The measurement accuracy of the system in this article was high, not only because of the high accuracy of sensor performance indicators, but also the following parameters were used in the measurement method. Due to the low setting speed of the drone, the average horizontal moving speed was $2 \mathrm{~m} / \mathrm{s}$, and the flying distance of the crop height range was 7.5 8 $\mathrm{m}$, which is only $1 / 11$ of the sensor measurement range, so the relative error of the system 
measurement accuracy was low ${ }^{[32]}$.

Table 2 System measurement and manual measurement of cotton plant height

\begin{tabular}{cccc}
\hline $\begin{array}{c}\text { Crop } \\
\text { numbers }\end{array}$ & $\begin{array}{c}\text { Manual } \\
\text { measurement/cm }\end{array}$ & $\begin{array}{c}\text { System } \\
\text { measurement/cm }\end{array}$ & $\begin{array}{c}\text { Relative error } \\
/ \%\end{array}$ \\
\hline h1 & $27.33 \pm 3.42$ & $23.85 \pm 5.55$ & -12.73 \\
h2 & $36.29 \pm 4.85$ & $33.29 \pm 6.65$ & -8.26 \\
h3 & $23.84 \pm 2.55$ & $22.92 \pm 5.39$ & -3.86 \\
\hline
\end{tabular}

Note: Data were expressed by means \pm standard deviation.

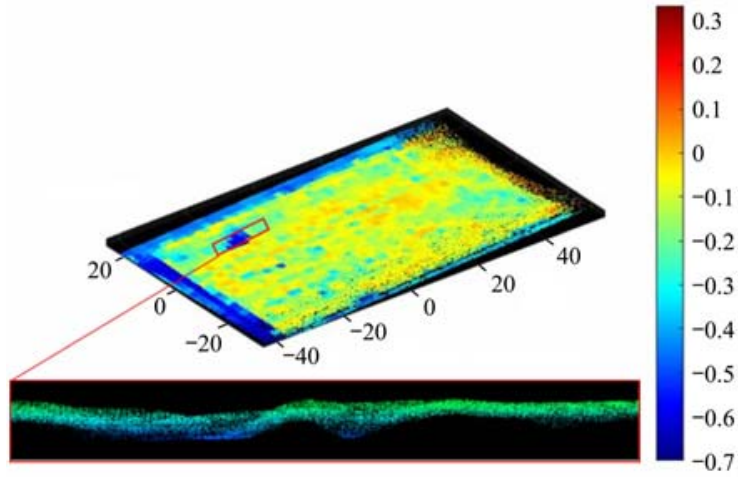

Figure 2 Cotton height map

\subsection{Cotton groups height coefficient of variation}

It has significant to reflect the height information of the cotton plant with respect to the geographical position and evaluate the height change, which can provide various operation information for agricultural machinery. This paper chooses the coefficient of variation to measure the change of crop groups height in the crop row or perpendicular to the crop row. Before statistical analysis, the point cloud was divided into a regional grid with a side of $2 \mathrm{~m}$. considers that the height of the point cloud in the grid is equivalent to the height of a group crops. The change of the height coefficient of variation along the crop row direction is shown in Figure 3.

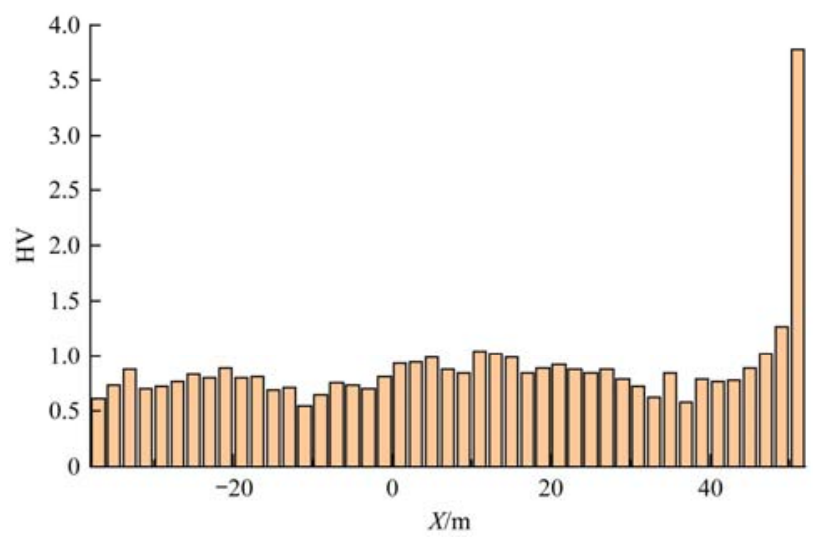

Figure 3 Distribution of height variation coefficient along the row direction $(X)$

Along the direction of crop rows, the coefficient of variation (CV) of height is between 0.54-1.04, the average coefficient of variation of height is 0.73 , and the abnormal value of the coefficient of variation occurs near $\mathrm{X}$ equal to 50 .

The length of the cotton field is about $220 \mathrm{~m}$ and the length of the sensor data collection area is $40 \mathrm{~m}$. There are few data sampling points in the non-test section of the field, and the degree of dispersion is too large, resulting in an abnormal coefficient of variation. The variation of the height coefficient of variation perpendicular to the crop row direction is shown in Figure 4. The crop height variation coefficient ranges from 0.06-1.27, and the average variation coefficient is 0.58. Comparing the coefficient of variation in the two directions, it can be concluded that the coefficient of variation of the geometric characteristics of the crop height shows a difference in the geographical location, and the coefficient of variation of the height along the crop row direction is larger. The results show that the height of the plant along the direction of the crop row may become the reference information for variable mechanical operations in the field.

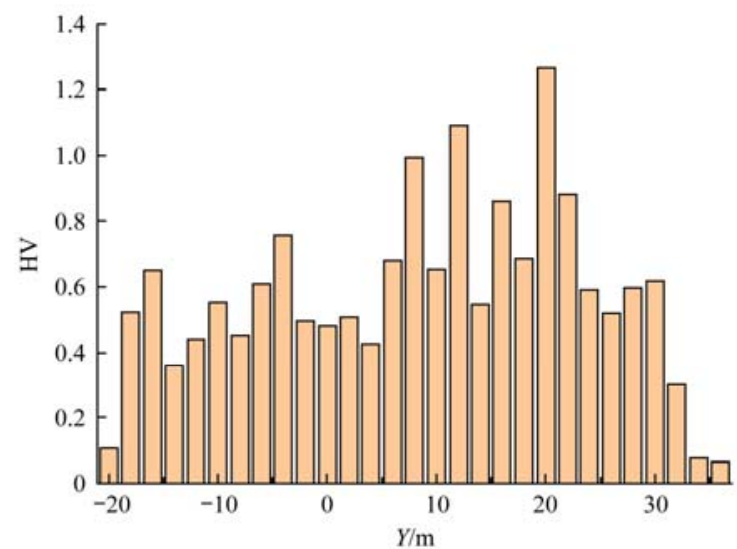

Figure 4 Vertical line direction $(Y)$ height variation coefficient distribution diagram

\section{Conclusions}

Differential information needed to be provided during cotton field mechanical variable operations. In order to obtain differential operation information, this paper studies the differences in the spatial distribution of cotton field plant heights.

1) This paper used the UAV-LiDAR detection system to obtain the point cloud data of the target cotton field. Stepped such as data preprocessing, extraction of target fields, calculation of crop height and spatial variability calculation, the spatial variability of cotton plant height with and perpendicular to the row direction of the crop was obtained.

2) The maximum relative error of the measured value of the UAV-LiDAR detection system was $12.73 \%$, and the corresponding maximum error is $3.48 \mathrm{~cm}$.

3) Along the direction of crop rows, the coefficient of variation (CV) of cotton plant height at the ranged between 0.54 and 1.04 , and the average coefficient of variation of height was 0.73 .

The results were shown that the height of the plant along the direction of the crop row may become the reference information for variable mechanical operations in the field. The research work of this article can provide reference for the information acquisition method of field variable machinery. Future work will automatically extract the point cloud of cotton field plants and import the generated spatial difference parameters into the map for variable mechanical access.

\section{Acknowledgments}

This work is carried out by the National Key Research and Development Plan (Grant No. 2017YFD0701005) and Key Research and Development Projects of Jiangsu Province (BE2017354). We also thank the anonymous reviewers for their critical comments and suggestions to improve the manuscript. We also thank the researcher Gong Yan of the Nanjing Institute of Agricultural Mechanization, Ministry of Agriculture and Rural Affairs for providing the drone for the experiment. 


\section{[References]}

[1] Thapa S, Zhu F Y, Walia H, et al. A novel LiDAR-based instrument for high-throughput, 3D measurement of morphological traits in maize and sorghum. Sensors, 2018, 18(4): 1187-1201. doi: 10.3390/s18041187.

[2] Pabuayon I L B, Sun Y, Guo W, et al. High-throughput phenotyping in cotton: a review. Journal of Cotton Research, 2019, 2(1): 18-27. doi: 10.1186/s42397-019-0035-0.

[3] Wilkerson G, Jones J, Boote K, et al. Modeling soybean growth for crop management. Transactions of the ASAE, 1983, 26(1): 63-73. doi: 10.13031/2013.33877

[4] Richardson C W Weather Simulation for Crop Management Models. Transactions of the ASAE, 1985, 28(5): 1602-1606. doi: 10.13031/2013.32484

[5] Qiu Q, Sun N, Bai H, et al. Field-based high-throughput phenotyping for Maize plant using 3D LiDAR point cloud generated with a "Phenomobile". Frontiers in plant science, 2019, 10(1): 554-569. doi: 10.3389/fpls.2019.00554.

[6] Jimenez-Berni J A, Deery D M, Rozas-Larraondo P, et al. High throughput determination of plant height, ground cover, and above-ground biomass in wheat with LiDAR. Frontiers in plant science, 2018, 9(1): 237-255. doi: 10.3389/fpls.2018.00237.

[7] Blancon J, Dutartre D, Tixier M-H, et al. A high-throughput model-assisted method for phenotyping maize green leaf area index dynamics using unmanned aerial vehicle imagery. Frontiers in plant science, 2019, 10(1): 685-701. doi: 10.3389/fpls.2019.00685.

[8] Sun J, Cong S L, Mao H P, et al. CARS-ABC-SVR model for predicting leaf moisture of leaf-used lettuce based on hyperspectral. Transactions of the Chinese Society of Agricultural Engineering, 2017, 33(5): 178-184. doi: 10.11975/j.issn.1002-6819.2017.05.026.

[9] Haboudane D, Tremblay N, Miller J R, et al. Remote estimation of crop chlorophyll content using spectral indices derived from hyperspectral data. IEEE Transactions on Geoscience and Remote Sensing, 2008, 46(2): 423-437. doi: 10.1109/TGRS.2007.904836.

[10] Cammarano D, Fitzgerald G J, Casa R, et al. Assessing the robustness of vegetation indices to estimate wheat $\mathrm{N}$ in Mediterranean environments. Remote Sensing, 2014, 6(4): 2827-2844. doi: 10.3390/rs6042827.

[11] Mahan J R, Conaty W, Neilsen J, et al. Field performance in agricultural settings of a wireless temperature monitoring system based on a low-cost infrared sensor. Computers and Electronics in Agriculture, 2010, 71(2): 176-181. doi: 10.1016/j.compag.2010.0.

[12] Whitaker R T A level-set approach to 3D reconstruction from range data. International journal of computer vision, 1998, 29(3): 203-231. doi: 10.1023/A:1008036829907.

[13] Bietresato M, Carabin G, Vidoni R, et al. Evaluation of a LiDAR-based 3D-stereoscopic vision system for crop-monitoring applications. Computers and Electronics in Agriculture, 2016, 124(1): 1-13. doi: 10.1016/j.compag.2016.03.017.

[14] D. Tumbo S, Salyani M, D. Whitney J, et al. Investigation of laser and ultrasonic ranging sensors for measurements of citrus canopy volume. Applied Engineering in Agriculture, 2002, 18(3): $367 . \quad$ doi: 10.13031/2013.8587.

[15] Wei J T, Salyani M. Development of a laser scanner for measuring tree canopy characteristics: Phase 1. Prototype development. Transactions of the ASAE, 2004, 47(6): 2101-2107. doi: 10.13031/2013.17795.

[16] Wei J T, Salyani M. Development of a laser scanner for measuring tree canopy characteristics: Phase 2 . Foliage density measurement.
Transactions of the ASAE, 2005, 48(4): 1595-1601. doi: 10.13031/2013.19174.

[17] [17] Rosell Polo J R, Sanz Cortiella R, Llorens Calveras J, et al. A tractor-mounted scanning LIDAR for the non-destructive measurement of vegetative volume and surface area of tree-row plantations: A comparison with conventional destructive measurements. Biosystems Engineering, 2009, 102(2): 128-134. doi: 10.1016/j.biosystemseng.2008.10.009.

[18] del-Moral-Martínez I, Arnó J, Sanz R, et al. Georeferenced scanning system to estimate the leaf wall area in tree crops. Sensors, 2015, 15(4): 8382-8405. doi: 10.3390/s150408382.

[19] Shi Y, Thomasson J A, Murray S C, et al. Unmanned aerial vehicles for high-throughput phenotyping and agronomic research. PloS one, 2016, 11(7): e0159781. doi: 10.1371/journal.pone.0159781.

[20] Colaço A F, Trevisan R G, Molin J P, et al. A method to obtain orange crop geometry information using a mobile terrestrial laser scanner and 3D modeling. Remote Sensing, 2017, 9(8): 763. doi: 10.3390/rs9080763.

[21] Lei L, Qiu C, Li Z, et al. Effect of leaf occlusion on leaf area index inversion of maize using UAV-LiDAR data. Remote Sensing, 2019, 11(9): 1067-1082. doi: 10.3390/rs11091067.

[22] Jin S, Su Y, Gao S, et al. Deep learning: individual maize segmentation from terrestrial lidar data using faster R-CNN and regional growth algorithms. Frontiers in plant science, 2018, 9(1): 866-876. doi: 10.3389/fpls.2018.00866.

[23] Sun S,Li C. In-field high throughput phenotyping and phenotype data analysis for cotton plant growth using LiDAR. 2017 ASABE Annual International Meeting, 2017: 1.

[24] Sun S, Li C,Paterson A H In-field high-throughput phenotyping of cotton plant height using LiDAR. Remote Sensing, 2017, 9(4): 377-398. doi: 10.3390/rs9040377.

[25] Yuan H, Bennett R S, Wang N, et al. Development of a peanut canopy measurement system using a ground-based lidar sensor. Frontiers in plant science, 2019, 10(1): 203-216. doi: 10.3389/fpls.2019.00203.

[26] Guo T, Fang Y, Cheng T, et al. Detection of wheat height using optimized multi-scan mode of LiDAR during the entire growth stages. Computers and Electronics in Agriculture, 2019, 165(104959). doi: 10.1016/j.compag.2019.104959.

[27] Cheng M, Cai Z J, Wang N, et al. System design for peanut canopy height information acquisition based on LiDAR. Transactions of the CSAE, 2019, 35(1): 180-187. doi: 10.11975/j.issn.1002-6819.2019.01.022. (in Chinese)

[28] Ashapure A, Jung J, Yeom J, et al. A novel framework to detect conventional tillage and no-tillage cropping system effect on cotton growth and development using multi-temporal UAS data. ISPRS Journal of Photogrammetry and Remote Sensing, 2019, 152 49-64. doi: 10.1016/j.isprsjprs.2019.04.003.

[29] Colaço A F, Molin J P,Rosell-Polo J R Spatial variability in commercial orange groves. Part 1: canopy volume and height. Precision Agriculture, 2019, 20(4): 788-804. doi: 10.1007/s11119-018-9612-3.

[30] Elmegreen B G The Initial Stellar Mass Function from Random Sampling in a Turbulent Fractal Clou. The Astrophysical Journal, 1997, 486(2): 944-954. doi: 10.1086/304562.

[31] Wang K, Guo H, Liu W, et al. Extraction method of pig body size measurement points based on rotation normalization of point cloud. Transactions of the CSAE, 2017, 33(1): 253-259. doi: 10.11975/j.issn.1002-6819.2017.z1.038. (in Chinese)

[32] Guan X P, Liu K, Qiu B J, et al. Extraction of geometric parameters of soybean canopy by airborne 3D laser scanning. Transactions of the CSAE, 2019, 35(23). doi: 10.11975/j.issn.1002-6819.2019.23.012. (in Chinese) 